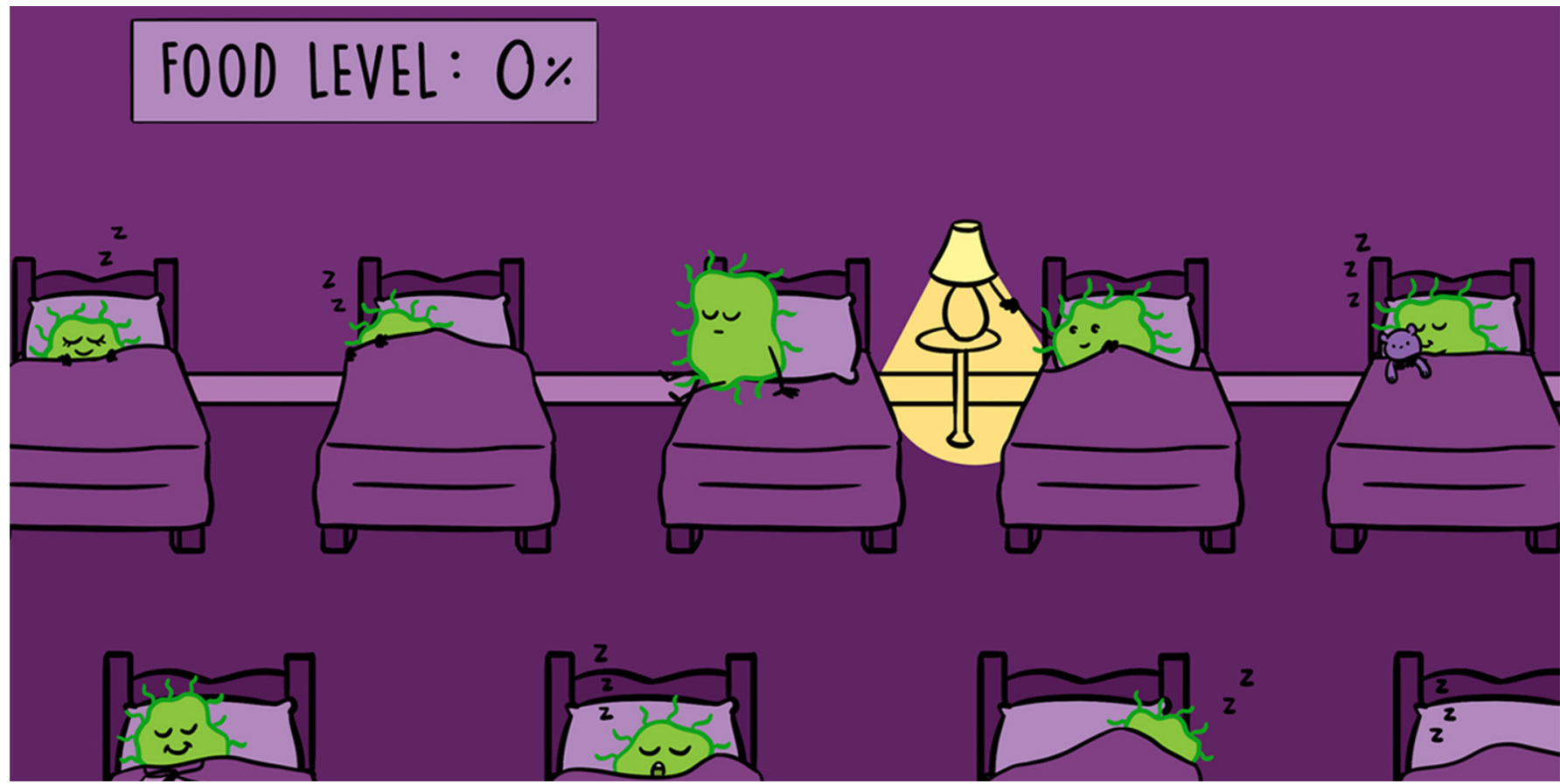

\title{
WHEN BACTERIA GO TO SLEEP
}

\section{Orit Gefen* and Nathalie Q. Balaban}

The Racah Institute of Physics, The Hebrew University of Jerusalem, Jerusalem, Israel

\section{YOUNG REVIEWERS:}

OHRTORAH STONE, AMIRIM PROGRAM

AGES: $12-13$

\section{BACTERIA}

Tiny creatures, unseen by the naked eye. Bacteria reproduce by cell division. Most bacteria are not harmful to humans, but some can cause disease.

When we say "bacteria," we usually think about tiny creatures, diseases, and the human body. So generally, we are thinking about the field of "biology." Therefore, people are usually surprised to find out that physicists, like us, also study bacteria. Physics is a scientific discipline that tries to describe the world accurately using mathematics, and physics studies different phenomena using quantitative analysis, which means accurate measurements. This is the reason why physics has a lot to offer other areas that might seem unrelated to it, like biology. In this article, we will talk about bacteria, and specifically about the battle with disease-causing bacteria and one interesting strategy that bacteria have developed in order to survive this battle, as understood through physics.

\section{HOW BACTERIA CHOOSE TO MULTIPLY OR TO GO DORMANT?}

Bacteria are very small creatures that are present all around us, within us, and almost everywhere. Bacteria are so small that they cannot be seen with the naked eye, but we can definitely see the influence of 
bacteria in our lives! For example, the bacteria that live in the gut help us to digest different substances; the bacteria that invade our food can ruin it; and some of the bacteria that enter our bodies might cause diseases-these are the bacteria that we want to fight.

To understand how we try to fight a community of billions of bacteria, first we need to understand how so many bacteria can be generated from one bacterium that enters the body. When bacteria are in the presence of food, for example inside the human body or in an animal's body, they eat and reproduce by cell division: one bacterium divides into two identical bacteria, those two bacteria eat and divide into four bacteria, those four bacteria divide into eight, and so on (see a cartoon of the bacterial reproduction process in Figure 1). Overall, the number of bacteria increases very quickly, at a rate that is called "exponential." Eventually, the available food always runs out and then the bacteria enter a state that is similar to sleep, where they try to use as little energy as possible, in order to have a chance to reproduce when food is available once again. This energy-saving state is called "dormancy," and we refer to these bacteria as "dormant." When a new source of food, such as sugar, arrives in the surroundings of a dormant bacterium, the bacterium acts almost like a sleepy child who needs to get up in the morning. Meaning, it takes a while for the bacterium to "wake up" and re-activate the essential systems for dividing, which it "turned off" in the dormant stage to save energy. At the end of the waking-up stage, the bacterium is again ready to start eating and dividing.

Figure 1

Exponential growth of bacteria. By the process of cell division, one bacterium divides into two identical bacteria, these two bacteria eat and divide into four bacteria, these four bacteria divide into eight, and so on. Eventually the food runs out and the bacteria become dormant.

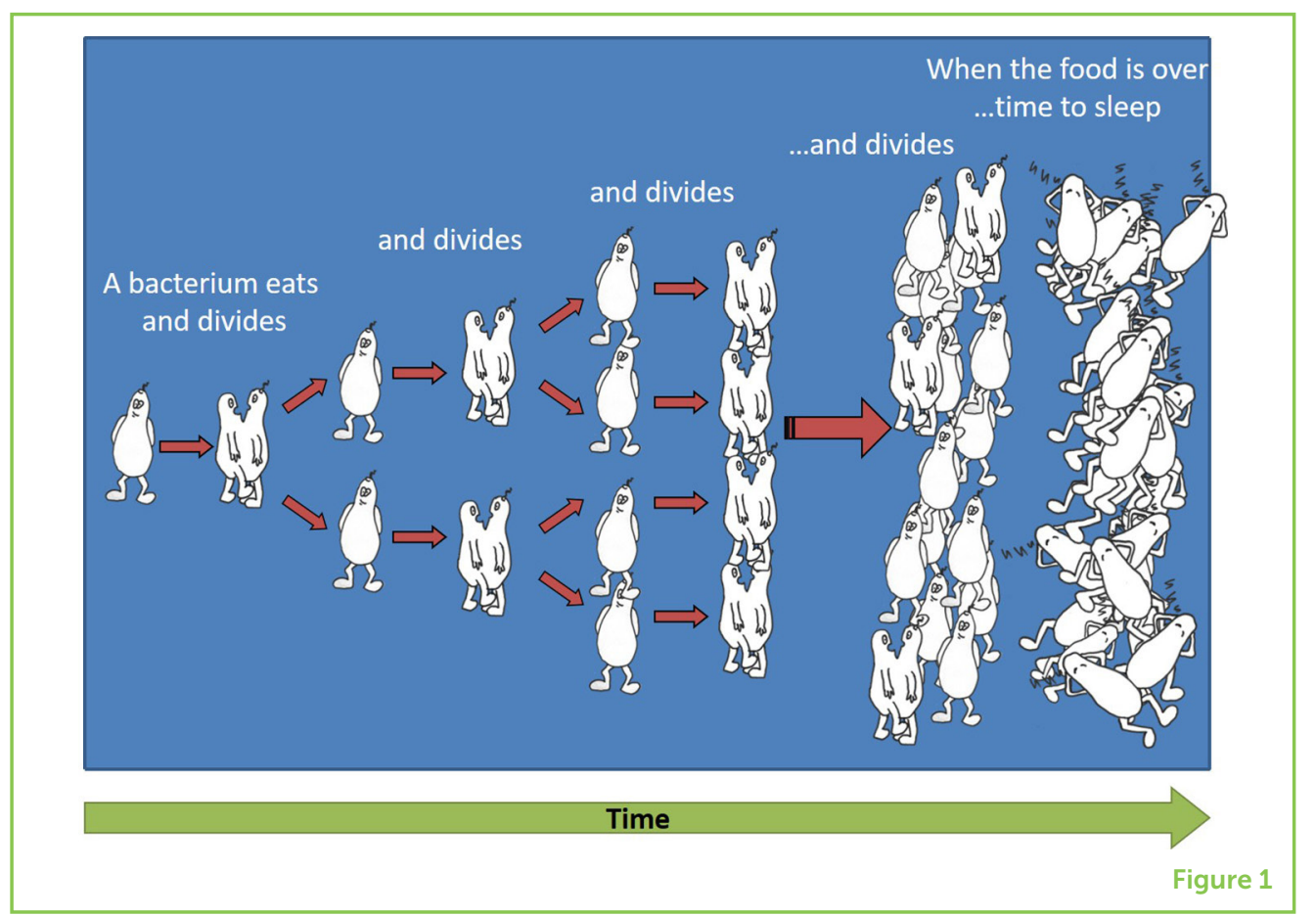




\section{ANTIBIOTICS}

Substances that are used as medicine against bacteria and are designed to harm the bacteria in a selective manner.

\section{SELECTIVE}

Acts in a specific way. Antibiotics are selective substances, because they are designed to kill bacteria, but they do not harm the cells of the body.

\section{THE ADVANTAGE OF BEING LAZY}

When pathogenic (disease-causing) bacteria invade the body, we need to fight them: our fabulous immune system can usually destroy the dangerous bacteria by itself, but there are cases where the immune system does not manage to do so, and then we get sick and sometimes need to use antibiotics. Antibiotics are substances that harm bacteria in a selective manner, meaning that they kill bacteria without harming the other cells that are around, namely the cells of the body.

Since the method of bacterial reproduction is different than the method used by cells of the human body, many antibiotics are designed to target the bacterial division mechanisms, and that makes the antibiotics selective-they harm bacteria but not human cells.

Sometimes we get sick, take antibiotics, and get better, but after a few days we feel sick again, and it turns out that we actually have the same disease. In order to understand how the same disease returns after antibiotic treatment, sometimes even more than once, we conducted an experiment in the lab. By looking at bacteria outside the body, in the lab, we can often begin to understand how the bacteria behave inside the body, as well. In our experiment, we gave the bacteria some food and saw that they divided and reproduced. Then, we added an antibiotic substance, mimicking antibiotic treatment, and observed what happened using a microscope.

We found something interesting and surprising: a small portion of bacteria stayed dormant while most of the bacteria grew. This means that when we gave the bacteria food and created good conditions for reproduction, most of them started to wake up and, as expected, eat and divide. However, a very small number of the bacteria stayed dormant. These dormant bacteria woke up much later than the rest. If food is still available, these late wakens will start eating and reproducing normally, like the ones that woke up earlier [1].

How do you think this experiment helped us understand the return of disease after antibiotic treatment ends?

Remember we said that antibiotics are selective and harm only bacteria, not the cells of the body? And that many antibiotics are designed to attack the cell division mechanism, because bacterial cells divide differently from human cells? So, if there is a dormant bacterium when antibiotics arrive, what will happen to it? If it does not try to divide, it will not be harmed. This means that if a bacterium stays dormant, it will survive the antibiotic treatment. Figure 2 shows a "lazy" dormant bacterium waking up and starting to divide after new food arrives. 
Figure 2

Dormant bacteria survive antibiotic treatment. Some dormant bacteria wake up and start to divide after new food arrives. If an antibiotic comes around, it kills all the dividing bacteria and only the dormant bacteria survive.

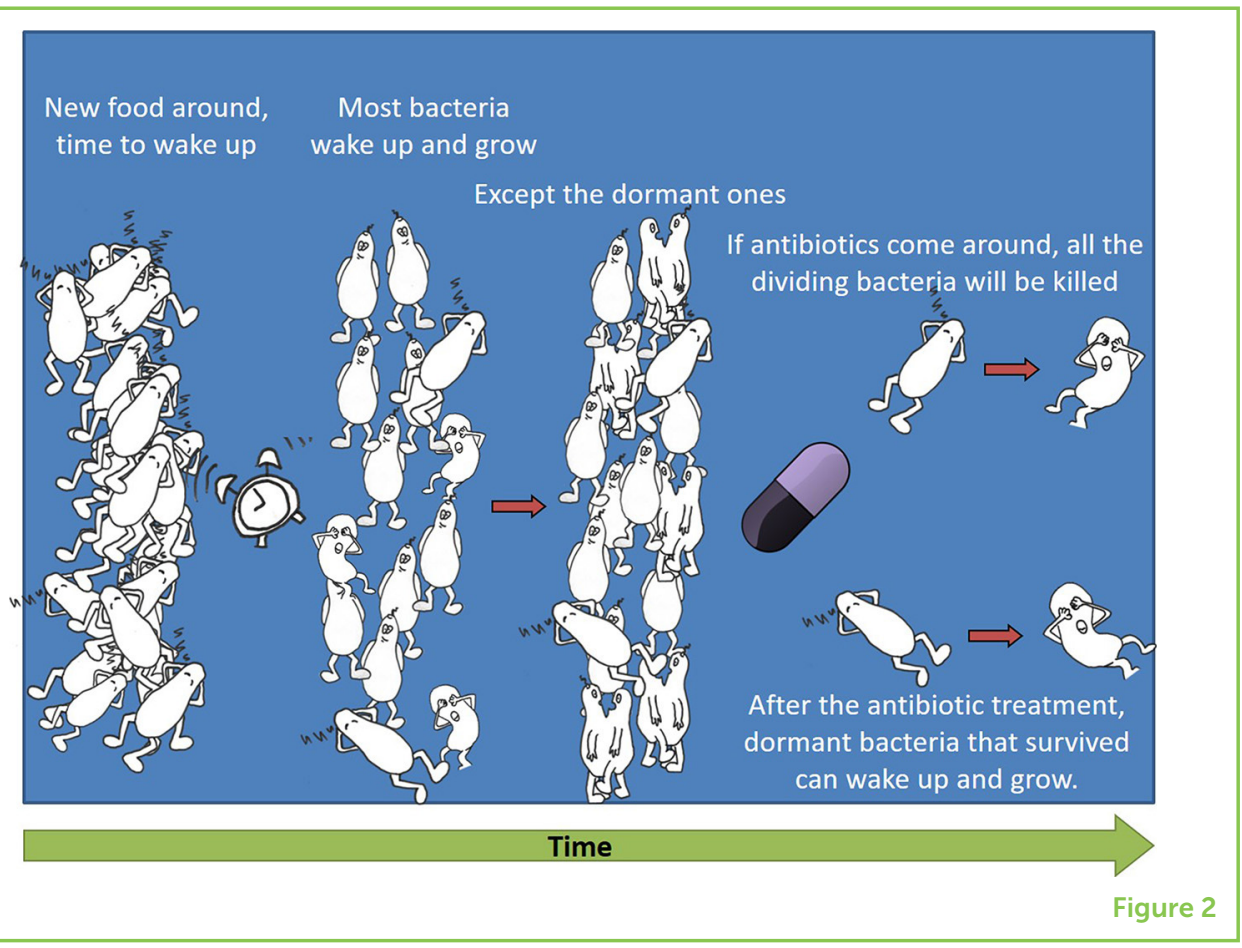

If an antibiotic comes around, it will kill all the dividing bacteria and only the dormant bacteria will survive.

If we get sick and take antibiotics, each bacterium that tries to divide dies. But, if there are a few dormant bacteria that sleep for a long while and do not wake up during the treatment, they will survive. If these bacteria wake up and start to divide after the antibiotic treatment is over, the disease might come back.

\section{BUILDING AN OBSERVATION DEVICE FOR BACTERIA UNDER ANTIBIOTIC TREATMENT}

In order to investigate this survival mechanism of bacteria, we built a special system called a "microfluidic" device, which is composed of three layers (see Figure 3). The first layer has tiny channels, inside which the bacteria are trapped at the beginning of the experiment. The second layer is a sort of thin skin (called a membrane) that has many tiny holes, like a strainer. This membrane is put on top of the bacteria in the channels. The holes in the membrane are smaller than the bacteria, so the bacteria cannot exit and they stay in the channels. In the third layer, there are more channels through which fluids can flow. The fluid can flow through the membrane to reach the bacteria. During the experiment, the fluid flowing in these top channels can be replaced, and in that way the environment of the bacteria in this microfluidic device can be changed. 


\section{Figure 3}

A microfluidic device for studying bacteria's survival mechanism

(A) The first layer of the system has very tiny channels, inside of which bacteria (shown in blue) are put at the beginning of the experiment.

(B) The second layer of the system is made of a membrane (yellow) with many tiny holes, like in a strainer. This membrane is placed on top of the bacteria, but they cannot move through the small holes. (C) The third layer of the system contains more channels through which fluids can flow. These fluids can move through the holes in the membrane to reach the bacteria. In this way, we can change the conditions the bacteria experience.

\section{INCUBATOR}

A chamber in which conditions, such as temperature and humidity are controlled. It is possible to grow bacteria in an incubator, at the proper temperature.
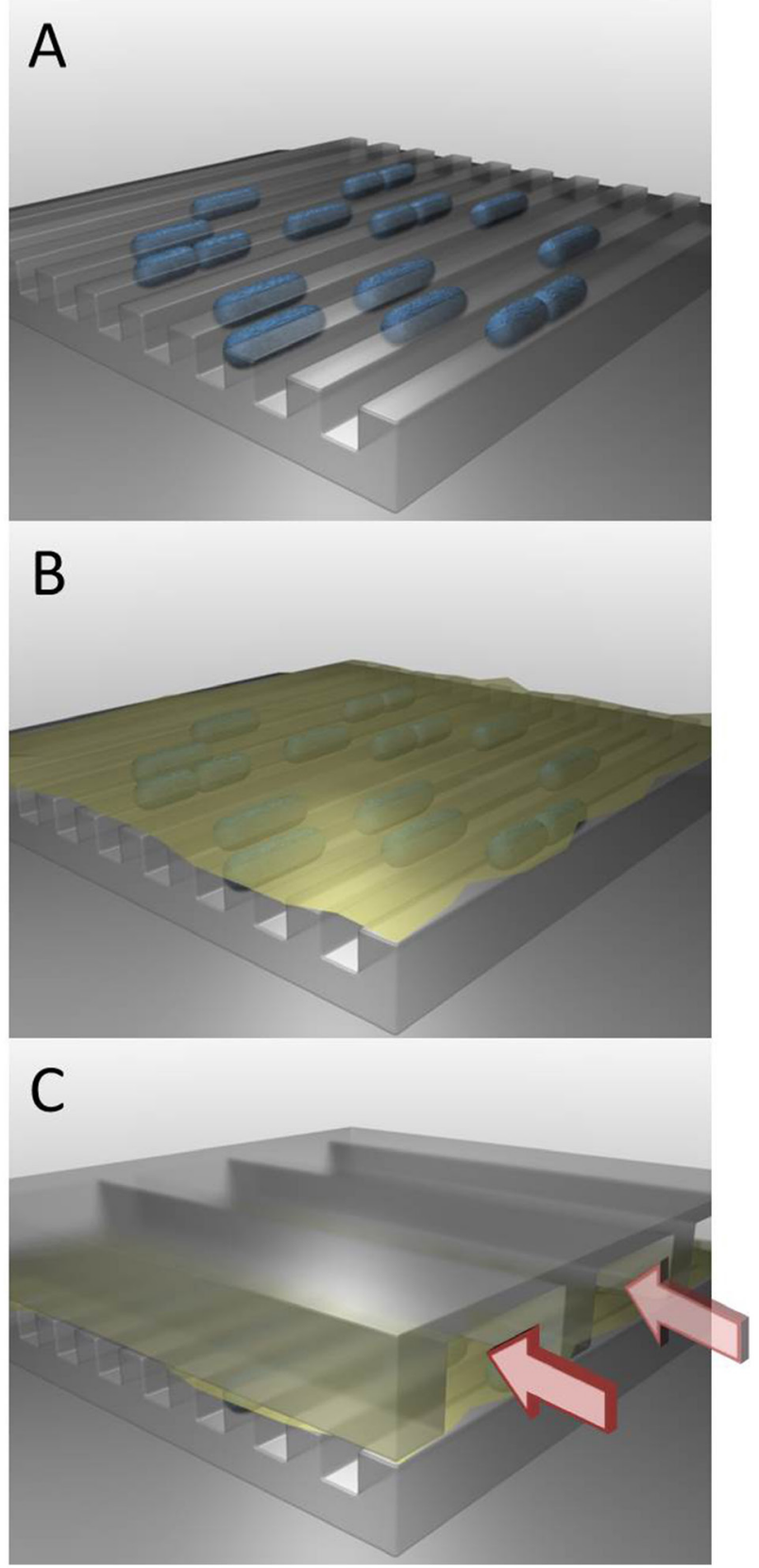

Figure 3

We placed this device under a microscope inside an incubator, which was heated to $37^{\circ} \mathrm{C}$, similar to the temperature inside the human body. The microscope was connected to a computer, so that the bacteria could be photographed and observed over time. At the beginning of the experiment (Figure 4A), we added a food-containing liquid to the top channels of the device. The food passed through the tiny holes in the membrane to reach the bacteria, and the bacteria woke up and started to grow (Figures 4B,C). Then, we passed a new fluid through the channels. The new fluid contained both food and antibiotics. The dividing bacteria died, as did every dormant bacterium that woke up during this stage of antibiotic treatment (Figures $4 D, E$ ). The dormant 
Figure 4

An image of real bacteria during the described experiment. The bacteria look light blue at the beginning of the experiment, and when they divide, they are shown in green. The bacteria have a slightly elongated shape and they are separate from one another. (A) A row of bacteria inside the microfluidic channels All the bacteria are blue (dormant). (B,C) Food is added to the upper channels and flows through the membrane to reach the bacteria. Some of the bacteria start growing and dividing, which you can see in green, but some bacteria stay dormant, like the one marked by the red arrow. (D,E) An antibiotic is added into the system, killing all the dividing bacteria. The only survivors are the ones who stayed dormant during the whole treatment. (F) After the antibiotic is washed away, some of the dormant bacteria start to wake up and divide. $(G, H)$ A new population of bacteria is produced from the two dormant bacteria that survived the antibiotic treatment
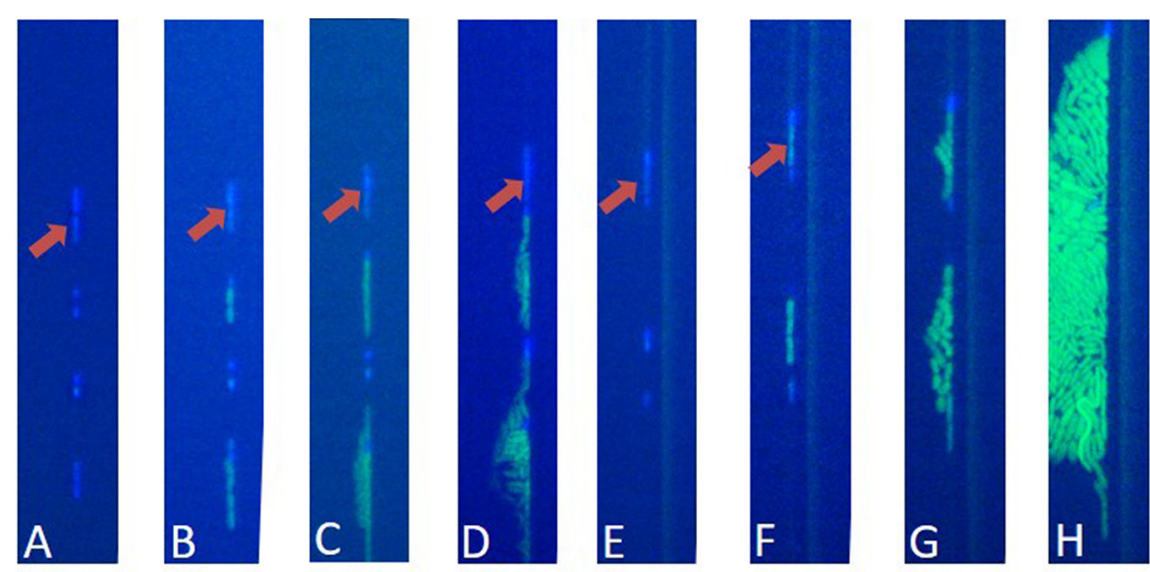

\section{In channels: food} antibiotic

\section{food}

Figure 4

bacteria, like the one marked with an arrow in the figure, survived. In the last stage, in order to mimic the end of the antibiotic treatment, we returned the food-containing fluid (without antibiotics) and saw that, some of the bacteria that were dormant and survived during the treatment period managed to wake up, divide, and create a new population of bacteria (Figures $4 \mathrm{~F}-\mathrm{H}$ ).

\section{UNDERSTANDING BACTERIAL STRATEGIES}

It is interesting to try to understand why bacteria behave this way. All the bacteria in the experiment (and usually also all the bacteria in an infection inside the body) came from one bacterium that divided many times, and therefore they should all be very similar. So, even though the bacteria are genetically identical and live in the same conditions, they have different behavior. What could the reason be for this? Since a bacterium wants to divide as much as possible to create as many offspring as possible, we might assume that, when conditions are good, all of the bacteria will immediately wake up and start dividing. But the results of our experiment showed that the bacterial population is made out of a big group of active, dividing bacteria, and a small group of dormant bacteria. In order to understand why there are two different groups of bacteria, active and dormant, let us think about the advantage of each state. We already mentioned the advantage that a dividing bacterium has over a dormant bacterium - it will have more offspring. But what is the advantage of a dormant bacterium over a dividing bacterium? The experiment we described demonstrates the advantage of a dormant bacterium -in the presence of antibiotics, the dormant bacterium will survive, and the growing bacterium will die. It turns out that, in nature, 
STRESS CONDITION

A state with tough conditions. Bacteria under stress conditions are experiencing something in the environment that can easily harm them, such as high temperature, strong acidity, or the presence of antibiotics.

ANTIBIOTICRESISTANT BACTERIA

Bacteria that can multiply even under an antibiotic. Many types of bacteria today are resistant to some types of antibiotics. It is harder to treat a patient who is infected with antibiotic-resistant bacteria, since not all types of antibiotics will be effective against the infection. there are additional stress conditions (tough kinds of challenging conditions that bacteria have to deal with), in which a dormant bacterium has higher chance of surviving.

So, it turns out that a bacterial population has a good strategy for longterm survival under stress conditions and, at the same time, for reproducing as much as possible: most bacteria in the population grow and divide, and a few bacteria stay dormant, serving as a "reserve." If the conditions kill the dividing bacteria, for example when they are exposed to antibiotics, the dormant minority will wake up, and in this way the bacteria population will reestablish itself when the conditions improve.

Using mathematics, we discovered that the number of dormant bacteria in a whole population should depend on the conditions that the bacteria are living in. If the conditions are always good, there should be no need for dormant bacteria. However, if the conditions are tough most of the time and there is a high chance of the bacteria dying, it will be better to have many dormant bacteria that slept most of the time and only occasionally wake up [2]. In nature, conditions are mostly good and allow for bacterial growth. Since extremely stressful conditions (like antibiotics) rarely occur in nature, it is best for most bacteria to keep dividing, with only a small percentage staying dormant as a "reserve" for emergency times. In order to confirm this physical model, we conducted additional experiments with bacteria in the lab and created different "living environments," with changing conditions. We saw that the bacterial populations changed and, in each population, the proportion of dormant bacteria at the end of the process fit the conditions in which they lived. A bacterial population that was exposed to antibiotics many times, and for long periods, changed so that the number of dormant bacteria was much higher than in a normal bacterial population, and those dormant bacteria only woke up after a very long time. A second bacterial population that was exposed to antibiotics for shorter time periods also changed to a population with many dormant bacteria, but the dormant bacteria woke up after a shorter time [3].

In other experiments, which we will not describe here, we showed that antibiotic-resistant bacteria are created more easily in a population of dormant bacteria [4]. The research that has been done so far tells us that in order to prevent the formation of antibiotic-resistant bacteria, we may use antibiotics that are capable of killing dormant bacteria, or add substances that wake up the bacteria along with the antibiotic treatment. These methods might be important in improving antibiotic treatment given to patients, to help patients avoid recurring infections and antibiotic-resistant bacteria. 
We are left with another interesting question-how does a bacterium "know" if it should wake up and start dividing, or whether it should stay dormant and serve as a "reserve" for emergency times? But this is a different story, and you are welcome to try to think about it yourself.

\section{ACKNOWLEDGMENTS}

We thank Naama Gefen for the cartoons.

\section{REFERENCES}

1. Balaban, N. Q., Merrin, J., Chait, R., Kowalik, L., and Leibler, S. 2004. Bacterial persistence as a phenotypic switch. Science 305:1622-5. doi: 10.1126/ science.1099390

2. Kussell, E., Kishony, R., Balaban, N. Q., and Leibler, S. 2005. Bacterial persistence: a model of survival in changing environments. Genetics 169:1807-14. doi: 10.1534/genetics.104.035352

3. Fridman, O., Goldberg, A., Ronin, I., Shoresh, N., and Balaban, N. Q. 2014. Optimization of lag time underlies antibiotic tolerance in evolved bacterial populations. Nature 513:418-21. doi: 10.1038/nature13469

4. Levin-Reisman, I., Ronin, I., Gefen, O., Braniss, I., Shoresh, N., and Balaban, N. Q. 2017. Antibiotic tolerance facilitates the evolution of resistance. Science 355:826-30. doi: 10.1126/science.aaj2191

SUBMITTED: 10 February 2019; ACCEPTED: 26 February 2019; PUBLISHED ONLINE: 18 March 2019.

EDITED BY: Idan Segev, Hebrew University of Jerusalem, Israel

CITATION: Gefen O and Balaban NQ (2019) When Bacteria Go to Sleep. Front. Young Minds 7:45. doi: 10.3389/frym.2019.00045

CONFLICT OF INTEREST STATEMENT: The authors declare that the research was conducted in the absence of any commercial or financial relationships that could be construed as a potential conflict of interest.

COPYRIGHT () 2019 Gefen and Balaban. This is an open-access article distributed under the terms of the Creative Commons Attribution License (CC BY). The use, distribution or reproduction in other forums is permitted, provided the original author(s) and the copyright owner(s) are credited and that the original publication in this journal is cited, in accordance with accepted academic practice. No use, distribution or reproduction is permitted which does not comply with these terms. 

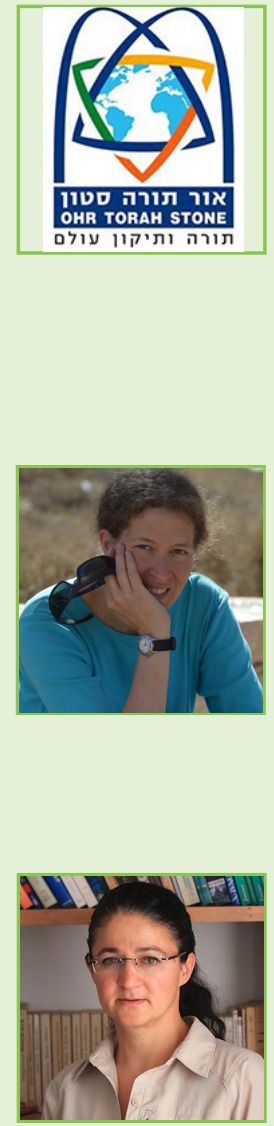

\section{YOUNG REVIEWERS}

\section{OHR TORAH STONE, AMIRIM PROGRAM, AGES: 12-13}

Seventh grade girls participating in the Amirim program for Excellence. Studying at Ohr Torah Stone in Jerusalem, a school that leads to values, creativity, and self-fulfillment for religious girls.

\section{AUTHORS}

\section{ORIT GEFEN}

I love to read, to think and write about interesting things, to dismantle everything I can and put it back together (and then find out what came out) and vanilla ice cream. I am investigating self-replicating systems at the Racah Institute of Physics, at the Hebrew University, a physics teacher at the Ohr Torah High School for girls in Jerusalem, and especially happy to know that there are so many things to learn and to dismantle in the world. *orit.gefen@mail.huji.ac.il

\section{NATHALIE Q. BALABAN}

The most fun part of science for me is when an observation that appears first as messy and incomprehensible turns out to be following a simple law. This is what Physics does again and again, and this is the fun we are after in biological systems. 